\title{
Mise en place syntectonique des minéralisations cuprifères du gîte d'Ifri (district du Haut Seksaoua, Haut Atlas occidental, Maroc)
}

\section{Syntectonic emplacement of the Ifri copper mineralization (High Seksaoua, western High Atlas, Morocco)}

\author{
Abdelaziz Gaouzi $^{\text {a, b}}$, Alain Chauvet ${ }^{c}$, Luc Barbanson', Lakhlifi Badra ${ }^{\text {a }}$, Jean-Claude \\ Touray $^{c}$, Saïd Oukarou ${ }^{\mathrm{d}}$ and Mohamed El Wartiti ${ }^{\mathrm{b}}$ \\ ${ }^{a}$ Faculté des sciences, université Moulay-Ismaïl, BP 4010 Beni-M'Hamed, Meknès, Maroc \\ ${ }^{\mathrm{b}}$ Faculté des sciences, université Mohamed-V, BP 1014, Rabat, Maroc \\ c ISTO, UMR 6113, université d'Orléans, bât. Géosciences, BP 6759, 45067 Orléans cedex 2, \\ France \\ ${ }^{\mathrm{d}}$ Snarema, 300, rue Mostapha-L.-Maâni, Casablanca 01, Maroc
}

\section{Résumé}

L'étude structurale et métallogénique (gîtologique) du gisement de cuivre d'Ifri (Haut Atlas occidental, Maroc) montre qu'une grande partie de la minéralisation est liée à un stockwerk, dont la formation est contrôlée par un cisaillement ductile tardi-hercynien à vergence NNW. Ce résultat significatif remet en question le caractère syngénétique jusqu'alors évoqué pour la minéralisation et permet ainsi de proposer un nouveau guide d'exploration pour ce type de gisement.

\section{Abstract}

Structural and metallogenic studies of the Ifri copper deposit (western High Atlas, Morocco) demonstrate that a great part of the mineralization was linked to a stockwork developed during a Late-Variscan NNW-verging shearing event. With this significant result, the until now accepted syngenetic character for this mineralization has to be reconsidered and allows to suggest a new guide of prospection for this kind of deposit.

Mots-clé : copper; stockwork; shearing; Late Hercynian; High Atlas; MoroccoMots-clé: cuivre; stockwerk; cisaillement; Tardi-Hercynien; Haut Atlas; Maroc 


\section{Abridged version}

\section{Introduction}

The copper mineralization of the High Seksaoua district (western High Atlas, Morocco) has been considered as a syngenetic deposit compared to a volcanic massive sulphide one [6 and 12]. Our new structural and mineralogical data allow to suggest an alternative to this interpretation and to demonstrate that the essential copper concentration was realized during a Late-Hercynian tectonic stage.

\section{Geological setting}

The mineralised district of the Hercynian High Seksaoua area (western High-Atlas, Morocco; Figure 1 and Figure 1) is known for its barytine and copper mineralizations. Our study concerns the Ifri copper deposit localized within a domain limited by two major faults: the Sembal and Tassa ones (figure 1c). The lithological succession is marked by the alternation between schists and limestones. From the base to the bottom, we can distinguish a lower calcschist unit, a discontinuous layer of black schist and grey dolomite, an upper calc-schist unit with limestone intercalations, a pelitic sandstone unit and finally a sericito-schist one ( figure 2). These units are traversed by NE-SW trending doleritic dykes of Late Hercynian age [11]. The copper mineralization systematically occurs between the upper part of the lower calc-schist unit and the top of the dolomite. These units have been attributed by facies analogy to the Lower to Middle Cambrian [3, 13 and 15]. More recently, this age has been discussed on the basis of geochemical data and a Neoproterozoic age for sediments deformed during the Panafrican was the adopted solution [10]. We cannot in this work solve this controversy even if we prefer to assimilate the herein studied deformation to the Hercynian orogeny and the sedimentary units to the Cambrian, as previously suggested [3, 4 and 14]. Southeast of the study area, the Tichka granite ( figure $1 b$ ) has been emplaced during the Hercynian orogeny [5 and 8].

\section{Structural evolution}

\subsection{Major events}

Our works on the Ifri mine and its surroundings (figure 3a) demonstrate a complex structural evolution in which it would be difficult to precisely date the deformations, although a relative chronology has been established. A first ductile event $\mathrm{D}_{1}$ was responsible for the foliation (figure $4 a$ ) associated with a NE-SW trending stretching lineation (figures $1 c$ and $4 b$ ), related folds $\left(\mathrm{P}_{1}\right)$ and top-to-the-SW shear criteria (figure $\left.3 b\right)$. $\mathrm{D}_{2}$ Knee-folds, with $\mathrm{N} 20^{\circ} \mathrm{E}$ trending axes and systematic overturning towards the east, were secondly formed (figure $3 c$ ). These folds are particularly developed within the calc-schist levels and clearly affect the $\mathrm{D}_{1}$-related shear criteria. After a weakly developed east-west shortening event ( $\mathrm{D}_{3}$, figure $\left.4 a\right)$, the last tectonics seems related to a regional extension associated to the Sembal and Tassa normal faults and the emplacement of the east-west- to NW-SE-trending barytine veins during the Permo-Trias [12] or the Trias [7]. Numerous faults oriented NNE-SSW to NE-SW and exhibiting a post-barytine left-lateral motion are also present within the studied area. 


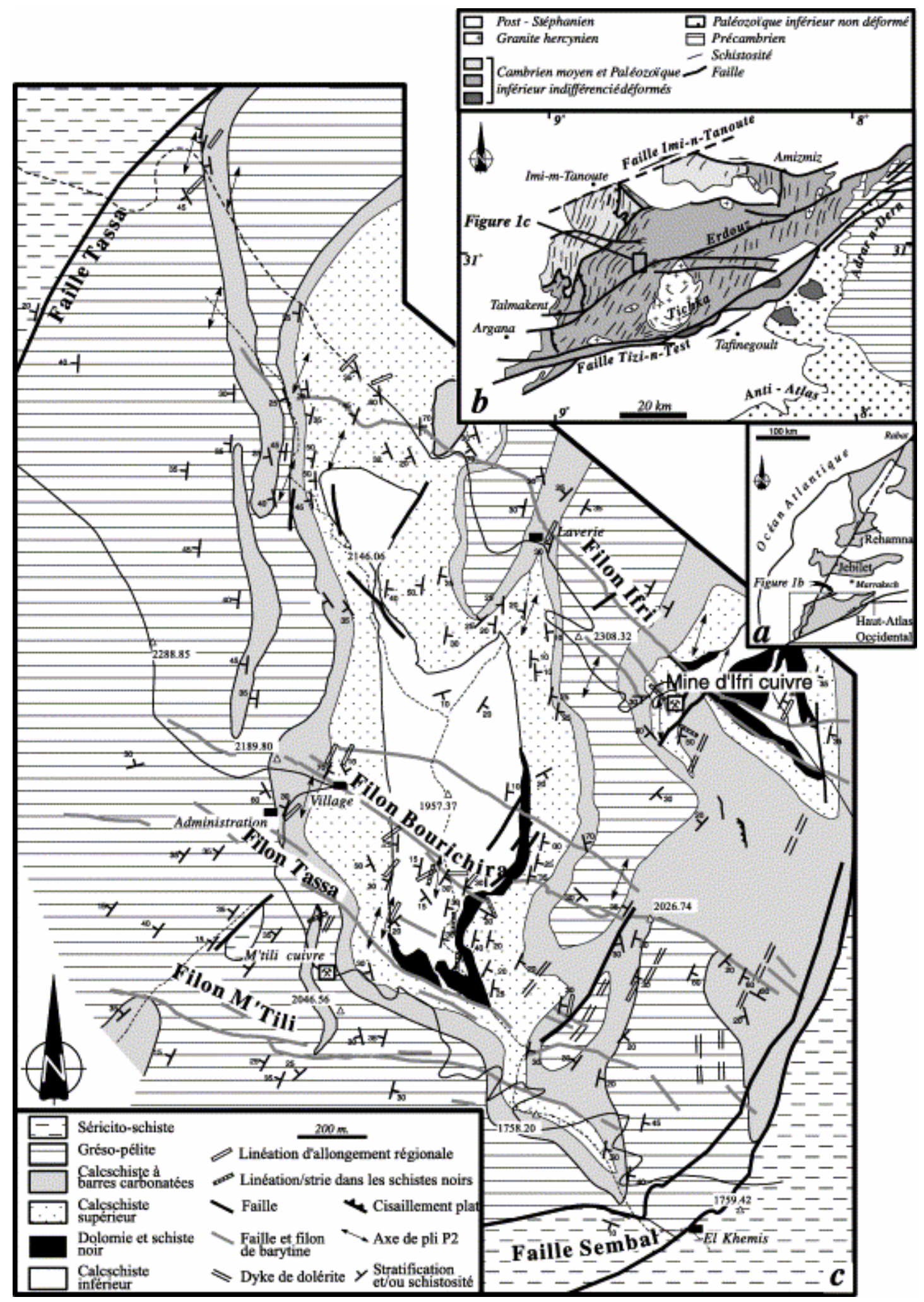

Figure 1. a. Localisation du Haut Atlas occidental dans les domaines hercyniens du Maroc central. b. Carte structurale simplifiée du Haut Atlas occidental, d'après Cornée et al. [3]. c. Carte structurale et géologique du Haut Seksaoua, entre les failles de Tassa et Sembal.

a. Location of the western High-Atlas within the Hercynian domains of Morocco. b. Simplified structural map of the western High Atlas after Cornée et al. [3]. c. Geological and structural map of the High Seksaoua district between the Tassa and Sembal faults. 


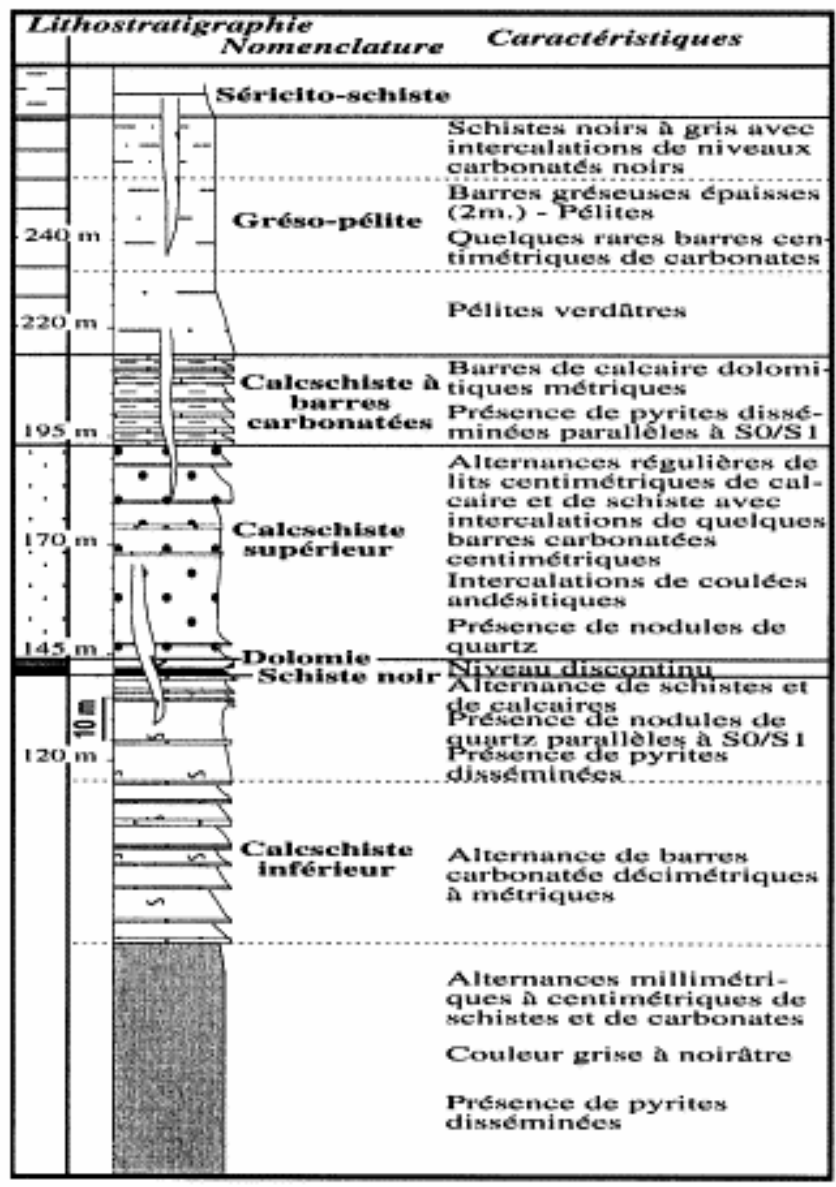

Figure 2. Succession lithostratigraphique du Haut Seksaoua.

Lithological succession of the High Seksaoua. 

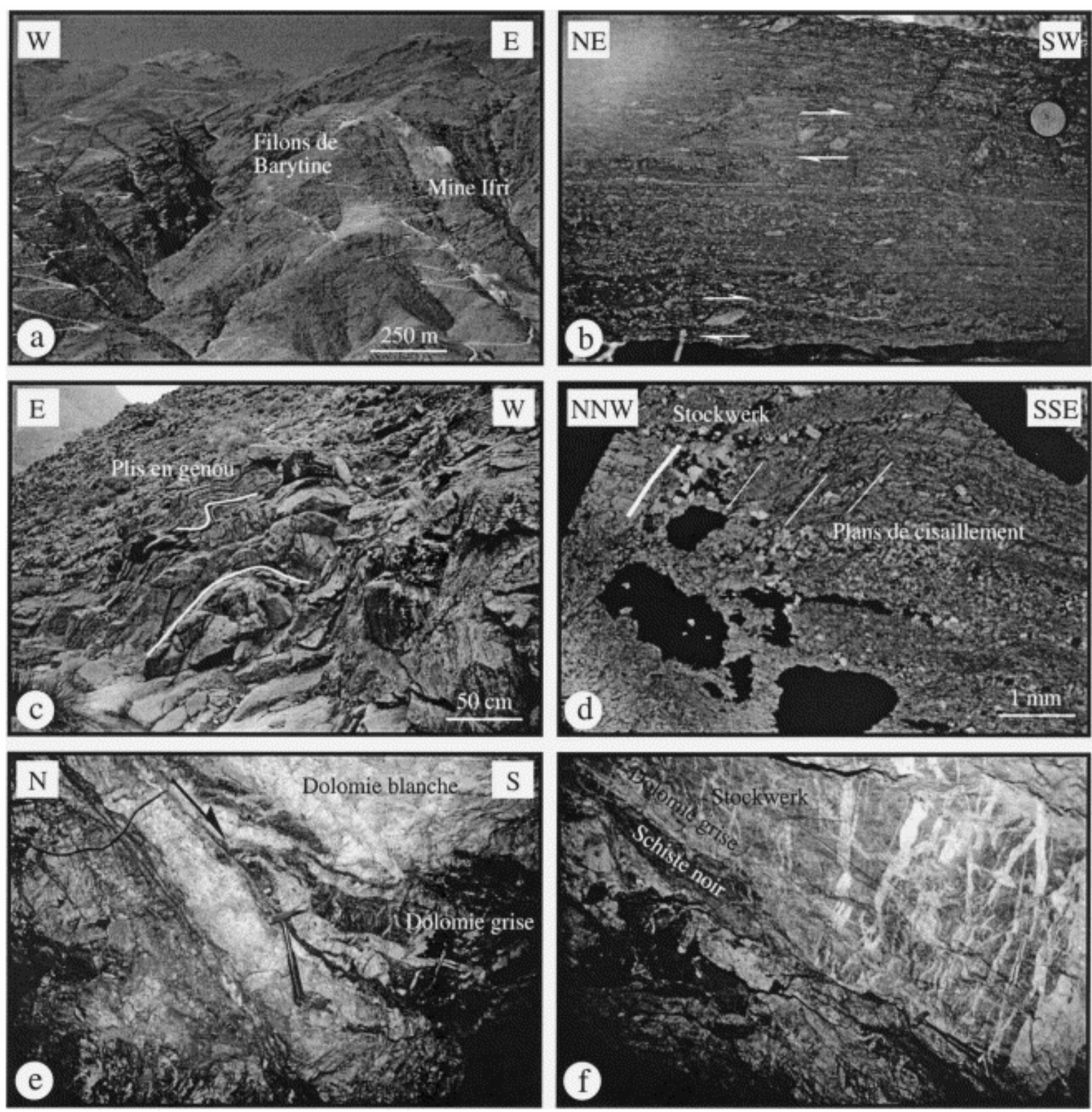

Figure 3. a. Vue d'ensemble du secteur étudié montrant la position de la mine de cuivre Ifri et celles des principaux filons de barytine. b. Critère de cisaillement $\mathrm{D}_{1}$ à vergence vers le sud-ouest. c. Géométrie en genou des plis $\mathrm{P}_{2}$ montrant un déversement systématique vers l'est. d. Parallélisme entre les veines du stockwerk et les plans de cisaillement ductile à vergence NNW dans les dolomies grises. e. Faille normale à vergence sud dans les alternances dolomie grise/schiste noir. f. Stockwerk minéralisé affectant un niveau de dolomie grise et accommodant la déformation ductile dans les schistes noirs.

a. General view of the studied area showing the location of the Ifri copper mine and the main barytine veins. b. $\mathrm{D}_{1}$ related SW-verging shear criteria. c. $\mathrm{D}_{2}$ related knee fold systematically overturned towards the east. d. Thin section showing the parallelism between stockwork veins and ductile top-to-the-NNW shear bands. e. South-verging normal faults affecting the dolomite and black schist levels. f. Mineralised stockwork developed within a dolomite level in response to the ductile deformation of the black schist. 


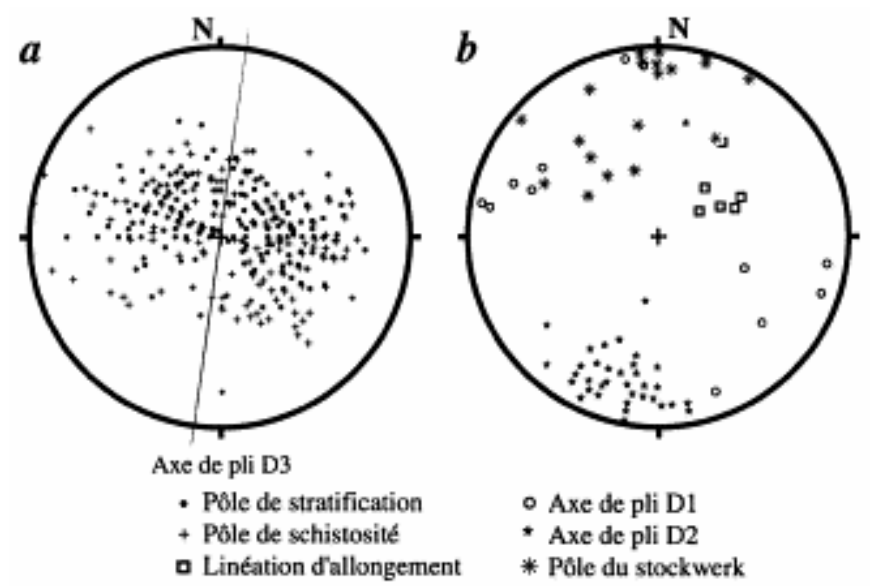

Figure 4. Distribution des différents éléments structuraux (diagrammes de Schmidt, hémisphère inférieur). a. Pôles des stratifications et des schistosités ; on remarque les effets des axes de plis $\mathrm{D}_{3}$. b. Linéations d'allongement, axes de pli $\mathrm{P}_{1}$ et $\mathrm{P}_{2}$ et pôles des fentes de tension représentant le stockwerk minéralisé.

Distribution of the structural elements (Schmidt diagrams, lower hemisphere). a. Poles of stratification and schistosity; we can notified the effects of the large-scale $\mathrm{D}_{3}$ fold. b. Stretching lineation $\mathrm{L}_{1}$ and fold axes $\left(\mathrm{P}_{1}\right.$ and $\mathrm{P}_{2}$ ) and pole of mineralised tension gashes (stockwork).

\subsection{The 'décollement' tectonics}

Within black schist and dolomitic levels, an additional tectonic event, characterized by top-tothe-NNW shear criteria (figure 3d), looks to be developed, thus explaining a relatively high rate of deformation within these levels. This tectonics is particularly well developed within the black schists that display a relative low competence. The stretching lineation responsible for this event remains hardly observable certainly because of the erase by the late extension from which the effects are detectable within mine outcrops ( figure 3e), associated to northsouth-trending striations ( figure 1c). However, the observation of thin sections realized within several directions allows to deduce a NNW sense of shearing for this 'décollement'type tectonics, from which the place within the regional structural evolution remains questionable.

Associated to the 'décollement' within the black schist, formation of NE-SW to east-west tension gashes (figure $4 b$ ) within more competent dolomite levels was observed ( figure $3 f$ ). These veins represent the ore bodies and are interpreted as a mineralised stockwork. Within a dolomitic sample collected within the level 1 of the Ifri underground mines, geometrical relationships between northwest-verging ductile shear bands and such stockwork demonstrate the contemporaneity between 'décollement' tectonics and vein formation. Effectively, shear bands are parallel to the stockwork and became denser at the vicinity of the vein (figure $3 d$ ). Other similar relations have been observed within the other Ifri galleries.

\section{The copper mineralization}

Three hydrothermal events have been recognized prior to the barytine formation [2]. First, massive pyrite emplaced during the sedimentation. Secondly, white dolomite developed by replacement of the grey one. This event is assumed to be the first manifestation of the epigenetic hydrothermalism. Finally, the mineralised stockwork formed within the more competent lithologies ( Figure 3 and Figure 3), such as the grey and white dolomites. From a 
mineralogical point of view, earlier sulphide levels are constituted by massive pyrite (I) and very scarce chalcopyrite inclusions, whereas the stockwork contains the main copper mineralization with, by order or abundance: chalcopyrite, pyrite (II), quartz, ankerite, siderite, galena, sphalerite, gersdorffite, ulmannite, arsenopyrite, brannerite, pyrrhotite, native bismuth, bismuthinite, stannite, sheelite and hessite. Cassiterite, wolframite and native silver are also recognized in very weak proportions. Because cassiterite occurs within the stockwork, it cannot be considered as an indicator of a massive sulphide deposit likely for Neves Corvo deposit [1 and 9]. As copper is mainly contained by chalcopyrite, the economic mineralization seems related to the stockwork rather than to earlier stratiform pyrite I levels.

\section{Conclusion}

It has been demonstrated during this work that the concentration of the copper mineralization was syntectonic and that the mineralised stockwork formed in response of a top-to-the-NNW shearing event. This ductile shearing appears well developed within the black schist levels and is accommodated within the more competent grey and white dolomite by the formation of tension-gashes assimilated to the stockwork. They are oriented $\mathrm{N} 50^{\circ} \mathrm{E}$ to $\mathrm{N} 90^{\circ} \mathrm{E}$ and represent the ore bodies. This model classifies the copper deposit of the High Seksaoua district as a beautiful example of structurally controlled mineralization.

Because it is only affected by later extensional features, the 'décollement' tectonics is assumed to develop at the end of the structural evolution of the studied area, in response to a NW-SE direction of shortening. This event could be reasonably correlated to the Late Hercynian tectonics responsible, in the same area, of the Tichka granite emplacement at ca $291 \pm 5 \mathrm{Ma}$, also under the control of a NW-SE shortening direction [5 and 8]. Indeed, the Tichka granite represents a good candidate to explain the origin of mineralised fluids. We thus propose that the mineralised stockwork of the Ifri copper mine was formed under the control of a Late Hercynian related NNW-verging shearing event. This important result re-asks the until now admitted syngenetic character for this mineralization [6 and 12] and allows to propose a new model of formation for this kind of deposit that could help to the exploration program. 


\section{Introduction}

Parce qu'elle est systématiquement localisée au contact entre deux unités lithologiques, la minéralisation cuprifère du district minier du Haut Seksaoua (Haut Atlas occidental, Maroc) a été jusqu'alors interprétée en termes de dépôt syngénétique de type amas sulfuré [6 and 12]. Les nouvelles données structurales et minéralogiques présentées dans ce travail favorisent l'hypothèse d'une minéralisation épigénétique, mise en place lors des derniers stades de l'histoire tectonique hercynienne de la région étudiée. Les relations géométriques très étroites établies entre la minéralisation et la tectonique nous permettent de proposer un nouveau modèle de formation pour cette minéralisation et donc de suggérer une alternative au modèle classique de minéralisation syngénétique.

\section{Cadre géologique}

Le district minier du Haut Seksaoua, situé dans le Haut Atlas occidental paléozoïque (figures $1 a$ et $1 b$ ), est réputé pour ses minéralisations de barytine et de cuivre. Si la barytine est toujours en cours d'exploitation par la société minière Snarema, la minéralisation cuprifère, travaillée du temps des Almohades (XII ${ }^{\mathrm{e}}-\mathrm{XIII}{ }^{\mathrm{e}}$ siècle), est actuellement en phase d'évaluation des réserves, dans l'objectif d'une exploitation intensive future. La minéralisation de cuivre, objet de cette étude, appartient au site d'Ifri, localisé au cœur d'un secteur géologique limité par deux grandes failles d'ampleur régionale : les failles de Tassa, au nord, et de Sembal, au sud (figure 1c). La succession lithostratigraphique détaillée de cette région montre, du bas vers le haut (figure 2) : des calcschistes inférieurs, un niveau discontinu de schistes noirs, un horizon de dolomie grise, des calcschistes supérieurs à intercalations de coulées et/ou sills andésitiques, des calcschistes à barres carbonatées, des gréso-pélites et, pour finir, des séricito-schistes. Les épaisseurs estimées de chaque unité sont indiquées dans le log stratigraphique de la figure 2. L'ensemble de ces unités est recoupé par des dykes doléritiques NE-SW, supposés d'âge Tardi-Hercynien [11]. La minéralisation cuprifère est systématiquement localisée entre le toit des calcschistes inférieurs et le toit des dolomies grises. Ces formations ont été attribuées au Cambrien inférieur ou moyen par analogie de faciès [3, 13 and 15]. Plus récemment, sur des arguments géochimiques, cet âge Cambrien a été discuté et ces roches ont été interprétées comme déposées au cours du Néoprotérozoïque et déformées au Panafricain [10]. Dans notre étude, aucun argument ne sera amené pour choisir entre les deux solutions proposées plus haut. Toutefois, nous verrons que les déformations qui affectent le Haut Seksaoua nous semblent plutôt comparables aux structures qui caractérisent l'orogenèse hercynienne au Maroc. Cette hypothèse est en accord avec les travaux antérieurs, qui ont attribué la déformation ductile qui affecte ce secteur du Haut Atlas occidental au cycle varisque et les unités sédimentaires au Cambrien [3, 4 and 14]. Au sud-est du secteur étudié, on rencontre le massif granitique du Tichka (figure $1 b$ ), dont la mise en place s'effectue pendant l'orogenèse hercynienne [5 and 8].

\section{3. Évolution structurale du secteur}

\section{1. Événements majeurs}

Nos travaux sur le district minier d'Ifri et ses environs (figure 3a) nous ont permis de mettre en évidence cinq types de structures, relatives à des événements tectoniques de natures 
différentes. Ainsi, l'évolution structurale du secteur apparaît très complexe, même si, au premier regard, les sédiments donnent l'impression d'un taux de déformation faible, puisque la stratification $S_{0}$ a pu être aisément identifiée en plusieurs endroits.

L'événement le plus précoce est responsable de la formation de la foliation (figure 4a) et d'une linéation d'allongement orientée NE-SW (figures $1 c$ et $4 b$ ). Des critères à vergence vers le sud-ouest sont systématiquement associés à cette linéation (figure $3 b$ ). La formation de bandes de cisaillement a été essentiellement reconnue. Des plis isoclinaux $\mathrm{P}_{1}$ à plan axial subhorizontal sont bien exprimés dans les niveaux carbonatés que l'on rencontre dans les calcschistes supérieurs. Cet événement précoce se développe à l'échelle régionale ; les structures qui lui correspondent sont reconnaissables sur l'ensemble du secteur étudié.

Le second événement est associé au développement de plis en genou d'échelle métrique, remarquablement bien exprimés sur le terrain (pli $\mathrm{P}_{2}$ ). Ces structures, très caractéristiques, se développent principalement dans les calcschistes inférieurs et supérieurs. Les axes sont orientés $\mathrm{N} 20^{\circ} \mathrm{E}$ (figure $4 b$ ) et le déversement apparent est systématiquement dirigé vers l'est (figure $3 c$ ). Les critères de cisaillement à vergence vers le sud-ouest, attribués au premier événement tectonique, sont affectés par ces plis.

Après une phase de serrage est-ouest de faible importance, essentiellement représentée par la formation de plis droits peu fermés d'échelle hectométrique (figure 4a), la région est affectée par une phase d'extension majeure, qui développe les failles normales NE-SW affectant le secteur (failles de Sembal et de Tassa, par exemple, figure 1c). Les filons de barytine, contrôlés par des failles normales orientées est-ouest à NW-SE, sont également interprétés comme étant contemporains de cet événement extensif. En effet, ces filons de barytine, à la fois coupés par les accidents de direction NE-SW (figure 1c) et sécants sur les injections de quartz de la faille Sembal, semblent ainsi être contemporains de ces structures. Sur des arguments d'ordre régional, cette extension a été interprétée comme étant d'âge PermoTriasique [12] ou Triasique [7]. De nombreuses failles, orientées NNE-SSW à NE-SW, se rencontrent également dans le secteur, et plus particulièrement dans la zone minéralisée d'Ifri. Elles présentent toutes un jeu senestre post-barytine.

L'ensemble des structures décrites ci-dessus, et tout particulièrement les failles, ont été réutilisées pendant les phases alpines, dont les effets restent tout de même assez faibles dans le secteur étudié.

\subsection{La tectonique en décollement}

Dans les niveaux de dolomie et de schistes noirs, il semble qu'un événement tectonique supplémentaire vienne s'ajouter ou s'intercaler aux précédents. Les roches de ces niveaux montrent un taux de déformation plus important que celles des autres unités. Des critères de cisaillement ductiles, dont la vergence est clairement vers le NNW, ont été observés (figure $3 d$ ). Ces critères sont très bien exprimés dans les schistes noirs, à cause de leur faible compétence. La linéation d'allongement indicatrice de la direction de transport de cet événement reste très difficile à déterminer. Des lames minces orientées ont ainsi été réalisées dans plusieurs directions et la sélection des orientations le long desquelles les critères sont les mieux exprimés nous a permis de déduire une direction de transport que nous pouvons assimiler à la linéation d'allongement. Ces critères sont le mieux représentés dans les sections NNW. Nous pensons que cette linéation d'allongement a pu ensuite être effacée par la phase d'extension permo-triasique évoquée ci-dessus, dont les effets sont remarquablement 
exprimés dans les schistes noirs. Une linéation strie nord-sud, observée sur les surfaces planes des schistes associés à de nombreux critères à vergence sud (figure $3 e$ ), de caractère plus cassant, est interprétée comme contemporaine de cette extension.

Dans les passés dolomitiques, des fentes de tension accommodent la déformation ductile qui affecte les schistes noirs (figure 3f). Ces fentes forment le stockwerk et sont orientées NE-SW à est-ouest (figure $4 b$ ). Elles représentent les corps minéralisés. La relation spatiale, au niveau du contact lithologique dolomie/schiste noir, entre cette déformation ductile et la minéralisation démontre le lien existant entre les deux. L'observation, dans un échantillon provenant du niveau 1, de relations géométriques qui prouvent la contemporanéité entre ces deux types de structures vient confirmer cette hypothèse. Dans cet échantillon, les plans de cisaillement ductiles à vergence vers le NNW sont parallèles aux veines du stockwerk et leur nombre augmente en s'approchant de la veine (figure 3d). Ces observations démontrent le caractère co-génétique entre le cisaillement ductile dans les schistes noirs et la formation du stockwerk. Des relations similaires ont pu être observées à l'échelle macroscopique sur les parois de cette même galerie.

\section{La minéralisation cuprifère}

L'évolution minéralogique et hydrothermale du secteur de la mine d'Ifri se définit par la succession de trois épisodes, qui précèdent la barytine [2].

La minéralisation pyriteuse, non économique, se caractérise par des niveaux stratiformes de pyrite, probablement d'origine syn-sédimentaire. Cette minéralisation est la plus précoce du secteur et se présente sous deux formes principales : disséminée dans les schistes noirs ou en bancs massifs au toit ou au mur de ces derniers.

Dans un deuxième temps, des amas de dolomie secondaire spathique (dolomie blanche) se forment au sommet des calcschistes inférieurs. Ces amas présentent une structuration interne complexe. Au cœur, la dolomie blanche constitue le ciment d'une brèche contenant des éléments de schiste noir, de calcschiste et de minéralisation pyriteuse précoce. En périphérie, elle se présente en remplissage de fissures sécantes sur les calcschistes inférieurs, les schistes noirs et la dolomie grise. Sa mise en place est interprétée comme représentative des premiers stades de l'hydrothermalisme.

La fin du processus voit le développement du stockwerk minéralisé (figures $3 d$ et $3 f$ ) dans la tranche lithologique allant de la dolomie grise à la partie supérieure des calc-schistes inférieurs. Ce stockwerk est bien représenté dans les zones de forte compétence, telles que les amas de dolomie blanche et la dolomie grise, comme nous l'avons décrit plus haut. Il recoupe clairement la dolomie blanche, tout comme l'ensemble des unités. Il représente à nos yeux la minéralisation économique.

D'un point de vue minéralogique, les niveaux de minéralisations précoces, anté-stockwerk, sont essentiellement constitués de pyrite massive (I) contenant de très rares inclusions de chalcopyrite. Inversement, le stockwerk présente une minéralogie plus riche, dans laquelle on trouve, par ordre d'abondance : chalcopyrite, pyrite (II), quartz, ankérite, sidérite, galène, sphalérite, gersdorffite, ullmannite, arsénopyrite, brannérite, pyrrhotite, bismuth natif, bismuthinite, emplectite, stannite, scheelite et hessite. La cassitérite, la wolframite, l'argent natif et l'or ont été également identifiés, en très petites quantités, dans des micro-fissures et/ou en micro-inclusions de la chalcopyrite. Il est important de noter que la cassitérite est associée 
au stockwerk et ne peut donc pas être considérée comme un marqueur de la présence d'un amas sulfuré, comme dans le cas du gisement de Neves Corvo [1 and 9]. La chalcopyrite est le minéral principal porteur du Cu. Elle peut apparaître sous la forme d'inclusions dans la pyrite II ou bien en grandes plages dans des fissures affectant ces mêmes pyrites II. Par conséquent, la minéralisation cuprifère est essentiellement contenue dans les veines du stockwerk.

\section{Conclusion}

\subsection{Processus de formation de la minéralisation cuprifère}

Un résultat fondamental de nos travaux sur le secteur du Haut Seksaoua réside dans la mise en évidence d'un stockwerk minéralisé, qui semble représenter la minéralisation économique. La formation de ce stockwerk cuprifère a été clairement expliquée, grâce à l'existence de la tectonique en décollement observée dans les schistes noirs. Les relations géométriques entre les bandes de cisaillement associées à cette tectonique et les veines du stockwerk (figure 3d) constituent une remarquable démonstration du caractère syntectonique de la minéralisation. Il est proposé que l'ouverture des veines du stockwerk soit le résultat de l'accommodation, dans les bancs dolomitiques plus compétents, de la tectonique ductile qui affecte les schistes noirs. Ainsi, l'orientation globale est-ouest à NE-SW des veines du stockwerk est en accord avec leur développement, en réponse à un cisaillement vers le NNW. Ce modèle fait des minéralisations cuprifères du Haut Seksaoua un très bel exemple de concentration syntectonique de la minéralisation et remet en question leur origine syngénétique, qui était souvent suggérée dans la littérature [6 and 12].

\subsection{Discussion}

Si l'évolution structurale du Haut Seksaoua apparaît complexe et si la formation des plis en genou, par exemple, reste encore inexpliquée, il est toutefois raisonnable de proposer que la minéralisation cuprifère de type stockwerk se forme lors d'un événement tectonique ductile, qui voit la formation de cisaillements à vergence NNW dans l'horizon de dolomie/schiste noir. Ce modèle n'exclut pas l'existence de minéralisations précoces syngénétiques dont l'intérêt économique n'a pour l'instant pas été démontré. La tectonique responsable de la mise en place de la minéralisation, associée à une direction de raccourcissement NW-SE, représente le dernier événement tectonique souple qui affecte le secteur étudié, puisqu'il est seulement affecté par les phénomènes extensifs tardifs (ce travail). Il peut donc être attribué, soit à la tectonique tardi-hercynienne, soit à la tectonique alpine. Il nous semble plus vraisemblable de corréler cet événement à la tectonique tardi-hercynienne, puisque cette période est celle de la mise en place du granite du Tichka, également sous l'effet d'une direction de raccourcissement NW-SE [8]. Le granite du Tichka étant daté à 291 \pm 5 Ma [5], nous proposons donc un âge Tardi-Carbonifère/Permien pour la minéralisation cuprifère d'Ifri. L'origine des fluides minéralisateurs est certainement à rechercher dans la mise en place voisine du granite du Tichka, hypothèse qui restera à confirmer par des investigations supplémentaires.

\section{Remerciements}

Les auteurs remercient les membres de la famille Lazrak (compagnie Snarema) et les responsables de la mine (A. Mahboub et B. Driss) pour l'accueil, les autorisations d'accès aux galeries de la mine Ifri Cuivre et leur confiance dans leurs travaux. J.-J. Cornée est remercié 
pour ses remarques constructives. L'Action intégrée franco-marocaine $n^{*} 1014 / 95$ a financé les missions de terrain.

\section{Bibliographie}

1. F. Aye and P. Picot, Sur les minéraux d'étain dans les amas sulfurés massifs : découvertes récentes, inventaire, gîtologie. C. R. Acad. Sci. Paris, série D 282 (1976), pp. 1909-1912.

2. L. Badra, L. Barbanson, A.M. Mechiche, J.-C. Touray, A. Chauvet and A. Gaouzi Histoire polyphasée du gisement de cuivre d'Ifri, Haut Atlas occidental (Maroc), $17^{\complement}$ RST (1998) p. 66

3. J.-J. Cornée, J. Ferrandini, J. Muller and B. Simon , Le Haut Atlas occidental paléozoïque : un graben cambrien moyen entre deux décrochements dextres $\mathrm{N}^{\circ} 0^{\circ} \mathrm{E}$ hercyniens (Maroc). $\mathrm{C}$. R. Acad. Sci. Paris, série II 305 (1987), pp. 499-503.

4. J.-J. Cornée Le Haut Atlas occidental paléozoïque : un reflet de l'histoire hercynienne du Maroc occidental, thèse, université Aix-Marseille-3 (1989) 901 p .

5. D. Gasquet Genèse d'un pluton composite hercynien. Le massif du Tichka (Haut Atlas occidental, Maroc), thèse, université de Nancy (1991) 413 p .

6. M. Hmeurras Étude gîtologique des minéralisations sulfurées du Haut Seksaoua, rapport, ministère de l'Énergie et des Mines, Rabat (1995).

7. L. Jaillard, Distribution des filons de barytine du Haut Atlas occidental. Géologie et Énergie, Rabat (1993), pp. 111-120.

8. J.-L. Lagarde and B. Roddaz, Le massif plutonique du Tichka (Haut Atlas occidental, Maroc) : un diapir syntectonique. Bull. Soc. géol. France 25 (1983), pp. 389-395.

9. X. Leca, L. Albouy, F. Aye and P. Picot, Caractéristiques principales du gisement de Neves-Corvo (Portugal). Chron. Rech. Min. 481 (1985), pp. 53-58.

10. H. Ouazzani, L. Badra, A. Pouclet and A.-E. Prost, Mise en évidence d'un volcanisme d'arc néoprotérozoïque dans le Haut Atlas occidental (Maroc). C. R. Acad. Sci. Paris, série IIa 327 (1998), pp. 449-456.

11. H. Ouazzani Le paléovolcanisme des secteurs de Guedmioua et du Haut-Seksaoua (massif ancien du Haut Atlas occidental, Maroc) : témoin d'un contexte convergent, thèse, université de Meknès (2000) 274 p .

12. S. Rchid Études géologiques et métallogéniques de la minéralisation de barytine et de cuivre du Haut Seksaoua (Haut Atlas occidental), thèse, université de Rabat (1996).

13. J.-P. Schaer, Volcanisme cambrien dans le massif ancien du Haut Atlas occidental. C. $R$. Acad. Sci. Paris, série D 258 (1964), pp. 2114-2117. 
14. M. Tayebi Étude géologique d'un tronçon du couloir de cisaillement hercynien de l'Ouest marocain dans le Haut Atlas occidental paléozoïque (Maroc), thèse, université Aix-Marseille3 (1989) 243 p .

15. H. Termier and G. Termier , Le Cambrien inférieur au voisinage du massif granitodioritique du Tichka (Haut Atlas marocain). C. R. Acad. Sci. Paris, série D 262 (1966), pp. 843-845. 